\title{
Health Related Quality of Life and Associated Factors among Epileptic Patients at Dessie Referral Hospital, Amhara Regional State, North East Ethiopia, 2020, A Cross Sectional Study.
}

Alebachew Sisay Stotaw ( $\sim$ dessmanaau2127@gmail.com )

Wollo University https://orcid.org/0000-0003-2491-6251

prem kumar prem

Wollo university

Dessale Abate Beyene

DBU: Debre Berhan University

Seid Legesse Hasen

Amhara Regional Agricultural Research Institute

Yimer Seid Ali

Amhara Regional Health Bureau: Amhara National Regional Health Bureau

\section{Research}

Keywords: Quality of life, Epilepsy, Ethiopia, Dessie

Posted Date: October 23rd, 2020

DOI: https://doi.org/10.21203/rs.3.rs-94451/v1

License: (c) (1) This work is licensed under a Creative Commons Attribution 4.0 International License.

Read Full License 


\section{Abstract}

Introduction: Quality of life is individuals' perceptions of their position in life in the context of the culture and value systems in which they live and about their goals, expectations, standards, and concerns. In the world, 70 million people suffer from epilepsy and $80 \%$ of epilepsy case was in the developing countries like Ethiopia. From a recent literature the prevalence quality of life in Ethiopia was 56.43. Due to different factor to contribute for the development of poor health-related quality of life that leads to the negative consequence on many patients with epilepsy; particularly in this study area, there were no studies done on the quality of life of epilepsy patients.

Objective: To assess health related quality of life among epileptic patients and associated factors at Dessie referral Hospital, Amhara regional state, North East Ethiopia, 2020.

Method: Facility-based cross-sectional study was conducted from April - June 2020. A systematic random sampling technique was employed to recruit a total of 415 study participants. Informed consent was taken for each participant and data was collected using the interviewer- administered questionnaire. Descriptive statistics was used to summarize the data while multivariate logistic regression analysis were employed to explore factors affecting the quality of life, $p \leq 0.05$ was considered as statistically significant.

Result: The response rate of this study was $92.5 \%$. Among the total study participant, $56 \%$ of them were male and their mean age of participant was 35.5 (SD \pm 14.4 ) years. Regarding occupation $33.3 \%$ of the participant was unemployed. Majority $95.8 \%$ of study participants had generalized seizure and $64.3 \%$ were taken two anti-epileptic drugs. From the total study participant, $12.2 \%$ and $37 \%$ were developing depression and anxiety respectively. The mean overall health related quality of life score of this study was 51.98 (95\% Cl: 41.9 - 62.06) out of 100. Age, educational status, marital status, occupation, residence, history of co-morbidity, family support and recreational activity was associated with health related quality of life $(p \leq 0.05)$

Conclusion: The mean overall quality of life for epileptic patients at Dessie referral hospital was low. Hence, a concerted effort must be done to improve patient quality of life towards the provided healthcare services.

\section{Introduction}

Epilepsy is a chronic condition that affects the quality of life (QOL) (1). It is a worldwide public health concern often accompanied by physical and cognitive disability leading to limitation in employment, independence, and social activities (2). Epileptic patients had significant impairment in their health related quality of life (3). Epilepsy is the most common neurological disorder that affects the patient's quality of life and their interactive social bond. In the world, 70 million people suffer from epilepsy and $80 \%$ Of cases occurred in the developing countries (4). In Ethiopia a recent cross-sectional study done at Ambo general hospital the mean value was 56.43 (5). 
Quality of life is defined as "individuals' perceptions of their position in life in the context of the culture and value systems in which they live and in relation to their goals, expectations, standards and concerns" (6). An individual's QOL can be physical, psychological, environmental, social \& spiritual domains of health that are influenced by a person's experiences, beliefs, expectations \& perceptions. Perception of life in the context of culture and values in relation to goals, expectations, and standards (1), (7), (8). QOL can be impaired by individuals living with epilepsy due to variety of reasons including complications of seizures, having a chronic health condition and social isolation (9).

Epilepsy causes significant psychological and social consequences for everyday living condition and the illness tends to have life time effects on the patient and the family (10). QOL in epileptic patients remains an important area of research and the assessment of quality of life should not be only focus on the evaluation of seizures but it must be include other life domains such as physical, environmental, cognitive, emotional, socio-occupational functioning, health perceptions and general satisfaction with life (10), (11). The issue of QOL was increasing to be considered as an important measure of how the disease affects patients' quality of life, especially for chronic diseases such as epilepsy (1). It affects life in multi-faced ways which should be studied in every community's cultural conditions and psycho social makeup. Shortness of medical services, unavailability of antiepileptic medications, and lack of awareness of medical treatment and cultural-related factors are common in developing countries including Ethiopia (2).

In developing countries epileptic patients had greater difficulties to attended school due to stigmatization that highly affects patient's quality of life (2). The degree of problem solving control and emotional subscale of quality of life are hidden their disease to their relatives and partners because of stigmatization (9). Many people in Africa believe that epilepsy is contagious and can be spread by urine, saliva, flatus, or feces excreted at all times or during a convulsion because of these unwilling to help or to touch the person who has fallen during a seizure lead to enormous social stigmas and lower QOL (3). A recent studies done in this hospital shows that $29.5 \%$ of epileptic patient had perceived their illness is not neurologic disorder rather they think it was a disease of spiritual concern (12).

The increased population in developing countries like Ethiopia affects the health system and adds socioeconomic burden requiring urgent attention. In this study area the hospital had served as a referral hospital for most north east Amhara region and Afar region. Patient's quality of life assessment must be done in this hospital because of epileptic patients are usually taking AEDs for long period of time that may affect the patient's quality of life. In this hospital one-third of the epileptic patients had experienced side effects and more than $30 \%$ of people with epilepsy do not attain full seizure control even with the best available treatment regimen because of poor adherence to AEDs (12).

In Ethiopia, few studies have been conducted on the health-related quality of life for epileptic patients so the disease become increase time to time as a result of ignorance (5). Hence, the purpose of the current study is to determine the health related quality of life and identify factors associated with quality of life among Epileptic patients at Dessie referral Hospital. 


\section{Methods Of Evaluation}

\section{Study design and period:-}

An institutional based cross-sectional study was conducted in Dessie referral hospital on epileptic outpatient department (OPD) from April -June 2020.

\section{Study area and setting:-}

Dessie referral Hospital is located in Dessie-City Administration, North East Amhara, Ethiopia. It was the only referral hospital in wollo and established in 1972. It serves as a training center for undergraduate and postgraduate medical students, pharmacists, nurses, midwives, medical laboratory technologists and radiologist. It is one the largest general public hospital. Almost all south \& north wollo zonal governmental hospitals, Afar region and private hospital use this hospital as referral unit and training sites. Dessie referral hospital serves about 500,000 patients per year in its outpatient department and about 40,000 in the inpatient and same number in the emergency department.

\section{Study and sources of population:-}

The study population was epileptic patients that were attending at psychiatry clinic of Dessie referral hospital during the study paired and all epileptic patients who were attended in Dessie referral hospital was the source of population.

\section{Inclusion and exclusion criteria of study:-}

All outpatients visiting psychiatry clinic of Dessie referral hospital with clinically confirmed diagnosis of epilepsy and having at least 6 months follow up at Dessie referral hospital and their age $\geq 18$ years was included in the study. On the other hand those patients who were mentally unstable such as aggressive patients, acute psychosis or panic attack and critically ill such as patients with status epileptic and any patient who acute sick is looking (determined by clinical presentation) were excluded in the study.

\section{Sample size determination and sampling technique:-}

Sample size was determined using the single proportion formula with assumption that overall HRQOL was $56.43 \%$ with margin of error $5 \%$, confidence level of $95 \%$. In Ethiopia; a recent cross sectional study done at Ambo general hospital the WHOQOL-BRIEF mean was 56.43 (5) and by adding $10 \%$ non-response rate a total of 415 study participants were recruited for the study. Systematic random sampling technique was employed to select the study subjects in Dessie Referral Hospital epileptic OPD. 


\section{Data collection tools and techniques:-}

Data was collected using data abstraction format and the standardized WHOQOL-BRIEF questionnaire. The data abstraction format was designed to extract information from medical chart and directly from the patient such as socio-demographic characteristics (age, gender, marital status, educational level, residence, and employment status, occupation ) as well as clinical characteristics (seizure type, frequency of seizure, number of AEDs and frequency of AED taken per day, history of any comorbid disease condition).

WHOQOL-BREF contains 26 items and a sound, cross culturally valid assessment of QOL, consisting of four domains (6): physical health (7 items), psychological health (6 items), social relationships (3 items), and environmental health (8 items); it also contains the first two questions on general perception of life and health and Each individual item of the WHOQOL-BREF were scored from 1(very dissatisfied/very poor) to 5 (very satisfied/very good).

HADS was a 14-item questionnaire, commonly used to screen for symptoms of anxiety and depression. The 14- items can be separated into two 7-item sub-scales for anxiety and depression(13).

\section{Data quality control/ Management:-}

This questionnaire which is developed in English was translated to Amharic and back to in English to check its consistency. Following that pre-testing was done in 10 selected epileptic patients at Boru Meda Hospital. Accordingly, all the necessary modifications and adjustments were done before implementing in the main study. In order to ensure its completeness and consistency, each data was checked by the principal investigator daily based in order to maintain the quality of the data.

\section{Data processing \& Analysis:-}

Data was analyzed by using SPSS version-21. Descriptive statistics including: frequency, mean and median was used to summarize socio-demographic, clinical and treatment related characteristics and evaluate distribution of responses. HRQOL scores was calculated using the WHO-QOL BREF tool (6). According to instruction manual, raw scores for the domains of WHOQOL-BREF were calculated and were transformed on the scale ranging from 0 to 100 by using mean transformed formula (mean transformed to $100=100 /$ (maximum score - no of items) $x$ (sum of means - no of items), where 100 the highest and 0 the lowest QOL (6). The mean score of each domain and the total score were also calculated since quality of life measures in studies are often presented as means. Therefore, categorization was done using the mean scores of WHOQOL-BREF. Subjects were categorized as having GOOD QOL in WHOQOL$B R E F$, those scores greater than or equal to mean While subjects with values less than the mean $(M)$, were categorized as having POOR QOL (3). In the HADs tool Subjects were categorized with the total score Of 0-7 was normal, a score of 8-10 ware borderline abnormal (borderline case) and a score of 11-21 were abnormal (case) (13). 
After checking the assumptions, multiple logistic regression analysis was performed to determine possible factors associated with quality of life. A bivariate analysis was conducted to obtain candidate variables for the multivariate regression models to determine possible predictors of outcome variable. In bivariate analysis, factors associated with quality of life with a $p$ value of $<0.2$ was considered a candidate for multivariate logistic regression model to identify strong factors associated with quality of life. $P$-value $<0.05$ was considered as statistically significant.

\section{Operational definition}

- Quality of life: - is defined as individuals' perceptions of their position in life in the context of the culture and value systems in which they live and in relation to their goals, expectations, standards and concerns (6).

- WHOQOL-BREF scale:- individual item was scored from 1(very dissatisfied/very poor) to 5 (very satisfied/very good) (6).

- Subjects were categorized as having GOOD QOL and POOR QOL based on their mean in WHOQOLBREF (3).

- GOOD QOL: - those scores values greater than or equal to mean of WHOQOL-BREF scale score.

- POOR QOL: - those scores values less than the mean of WHOQOL-BREF scale score.

- Based on HAD scale classified anxiety \&depression if the total score is(13):-

- 0-7 = Normal: - patients who have no any sign of depression and anxiety.

- 8-10 = Borderline abnormal (borderline case):- patients who have at borderline or have risk developing depression and anxiety.

- 11-21 = Abnormal (case):- patients who had sign or case of depression and anxiety

\section{Results}

\section{Socio-demographic characteristics of study participant}

The response rate of this study was $92.5 \%$. Among the total of 384 study participant, more than half of them were males $(215,56 \%)$. Regarding age distribution, the mean age of participant was $35.5(S D \pm 14.4)$ years; with $(111,28.9 \%)$ participants were in the age group of between $25-34$ years. A significant proportion of participant's ethnicity $(308,80.2 \%)$ was Amhara. Married $(176,45.6 \%)$ and Muslim (198, $51.6 \%)$ accounted the highest proportion of study participant. Regarding educational status, unable to read and write $(109,28.4 \%)$ accounted the highest proportion of the study participant. Highest proportion of the study participant $(128,33.3 \%)$ were unemployed and $(200,52.1 \%)$ were live in urban (Table-1). 
Table 1

Socio-demographic characteristics of study participant, Dessie referral hospital, Ethiopia, 2020

\begin{tabular}{|c|c|c|}
\hline Variables & Frequency & percentage \\
\hline \multicolumn{3}{|l|}{ Sex } \\
\hline Male & 215 & 56 \\
\hline Female & 169 & 44 \\
\hline \multicolumn{3}{|l|}{ Age in years } \\
\hline $18-24$ years & 105 & 27.3 \\
\hline 25-34 years & 111 & 28.9 \\
\hline $35-44$ years & 70 & 18.2 \\
\hline$>44$ years & 98 & 25.5 \\
\hline \multicolumn{3}{|l|}{ Religion } \\
\hline Muslim & 198 & 51.6 \\
\hline Orthodox & 142 & 37 \\
\hline Protestant & 44 & 11.5 \\
\hline \multicolumn{3}{|l|}{ Ethnicity } \\
\hline Amhara & 308 & 80.2 \\
\hline Tigre & 39 & 10.2 \\
\hline Oromo & 33 & 8.6 \\
\hline Afar & 4 & 1.0 \\
\hline \multicolumn{3}{|l|}{ Educational status } \\
\hline Unable to read and write & 109 & 28.4 \\
\hline Elementary & 98 & 25.5 \\
\hline Secondary & 76 & 19.8 \\
\hline Diploma & 52 & 13.5 \\
\hline Degree and Above & 49 & 12.8 \\
\hline \multicolumn{3}{|l|}{ Marital status } \\
\hline Single & 154 & 40.1 \\
\hline Married & 176 & 45.6 \\
\hline
\end{tabular}




\begin{tabular}{|c|c|c|}
\hline Divorced & 38 & 9.9 \\
\hline Widowed & 17 & 4.4 \\
\hline \multicolumn{3}{|l|}{ Occupation } \\
\hline Unemployed & 128 & 33.3 \\
\hline Famer & 50 & 13.0 \\
\hline House wife & 17 & 4.4 \\
\hline Employed & 102 & 26.6 \\
\hline Self-employed* & 87 & 22.7 \\
\hline \multicolumn{3}{|l|}{ Residence } \\
\hline Urban & 200 & 52.1 \\
\hline Rural & 184 & 47.9 \\
\hline
\end{tabular}

\section{Disease Related Characteristics and Treatment History of study participant}

Disease related characteristics and treatment history are depicted on Table-2. Majority $(368,95.8 \%)$ of the study participants had generalized seizure onset. Regarding the frequency of seizure $(359,95.8 \%)$ had more than one seizure episode per year and more than half $(247,64.3 \%)$ of study participants were taken two AEDs. 
Table 2

Disease Related Characteristics and Treatment History of study participant, Dessie referral hospital, Ethiopia, 2020

\begin{tabular}{|c|c|c|}
\hline Variables & Frequency & Percentage \\
\hline \multicolumn{3}{|l|}{ Seizure type } \\
\hline Generalized onset & 368 & 95.8 \\
\hline Focal onset & 16 & 4.2 \\
\hline \multicolumn{3}{|l|}{ Seizure frequency per year } \\
\hline >= one seizure per year & 359 & 93.5 \\
\hline Seizure free for year & 25 & 6.5 \\
\hline \multicolumn{3}{|c|}{ Number of anti-epileptic drugs taken per day } \\
\hline A single drug & 93 & 24.2 \\
\hline Two drug & 247 & 64.3 \\
\hline Three drug & 44 & 11.5 \\
\hline \multicolumn{3}{|l|}{ Duration of epilepsy in year } \\
\hline$<=5$ years & 64 & 16.7 \\
\hline $6-10$ years & 143 & 37.2 \\
\hline$>=11$ years & 177 & 46.1 \\
\hline \multicolumn{3}{|c|}{ History of Current comorbid illness } \\
\hline Yes & 50 & 13 \\
\hline No & 334 & 87 \\
\hline
\end{tabular}

\section{Personal characteristics of study participant}

Among a total of 384 study participants $(356,92.7 \%)$ had perceived self-esteem. Regarding compliance of self-care $(378,98.4 \%)$ had a capacity of self-care and most of study participant $(366,95.3 \%)$ had family support. Majority of study participant $(323,84.1 \%)$ had get enough and regular sleep. Regarding the participation of recreation activity $(263,68.5 \%)$ had the capacity of participating on recreational activity; on the other hand $(264,68.7 \%)$ were not doing any regular physical activity (Table-3) 
Table 3

Personal characteristics of study participant, Dessie referral hospital, Ethiopia, 2020

\begin{tabular}{|llll|}
\hline Variables & & Frequency & Percentage \\
\hline Perceived self esteem & Yes & 356 & 92.7 \\
\cline { 2 - 4 } Compliance to self-care & No & 28 & 7.3 \\
\cline { 2 - 4 } & Yes & 378 & 98.4 \\
\hline Family support & No & 6 & 1.6 \\
\hline Enough and regular sleep & Yes & 366 & 95.3 \\
\cline { 2 - 4 } & No & 18 & 4.7 \\
\hline Recreational activity & No & 61 & 84.1 \\
\cline { 2 - 4 } & Yes & 263 & 15.9 \\
\hline Regular physical activity & Yes & 120 & 68.5 \\
\cline { 2 - 4 } & No & 264 & 31.3 \\
\cline { 2 - 4 } & & 121 & 68.7 \\
\hline
\end{tabular}

\section{Hospital Anxiety and Depression Scale of study participant}

From the HADs scale stated on Figure-1. Most participants rated normal $(191,49.7 \%)$ followed by borderline depression $(146,38.0 \%)$ for depression subscale. On the other hand for anxiety subscale (164, $42.7 \%)$ were normal followed by $(142,37.0 \%)$ anxiety case.

\section{Mean score Health Related Quality of Life scale among patients treated for Epilepsy.}

Mean scores for the WHO-QOL BRIEF scales for the evaluation physical domain was 58.6, psychological domain mean was 50.25 , social domain mean was 53.3 and environmental domain mean was 48.75 , whereas rate of QOL mean was 43.75 and the satisfaction of their health condition mean was $47.75 \mathrm{In}$ general, the mean overall health related quality of life score was $51.98 \pm 10.08$ (Table-4). 
Table 4

Mean scores of Health Related Quality of Life scale among patients treated for Epilepsy, Dessie referral hospital, Ethiopia, 2020

\begin{tabular}{|c|c|c|c|}
\hline Variables & $\begin{array}{l}\text { Likert scale Mean } \\
\pm \text { SD }\end{array}$ & $\begin{array}{l}\text { Formula for domain } \\
\text { mean* }\end{array}$ & $\begin{array}{l}\text { Domain mean } \pm \text { SD } \\
\text { of } 100\end{array}$ \\
\hline Physical domain (7 items) & $3.34 \pm 0.47$ & $100 /(35-7) \times(23.4-7)$ & $58.6 \pm 3.3$ \\
\hline $\begin{array}{l}\text { Psychological domain } \\
\text { (6 items) }\end{array}$ & $3.0 \pm 0.47$ & $\begin{array}{l}100 /(30-6) \times(18.06- \\
6)\end{array}$ & $50.25 \pm 2.8$ \\
\hline Social domain (3 items) & $3.1 \pm 0.5$ & $100 /(15-3) \times(9.4-3)$ & $53.3 \pm 1.5$ \\
\hline Environmental (8 items) & $2.95 \pm 0.47$ & $100 /(40-8) \times(23.6-8)$ & $48.75 \pm 3.9$ \\
\hline How would you rate your QOL & $2.75 \pm 0.53$ & $100 /(5-1) \times(2.75-1)$ & $43.75 \pm 0.53$ \\
\hline $\begin{array}{l}\text { How satisfied are you with } \\
\text { your health }\end{array}$ & $2.91 \pm 0.55$ & $100 /(5-1) \times(2.91-1)$ & $47.75 \pm 0.55$ \\
\hline Overall HRQOL & $3.08 \pm 0.39$ & $\begin{array}{l}100 /(130-26) x \\
(80.06-26)\end{array}$ & $51.98 \pm 10.08$ \\
\hline
\end{tabular}

\section{Associated factors affecting health related quality of life}

In Bivariate analysis, among 22 investigated variables 16 of them showed $(P<0.2)$ association with quality of life measured using WHO-QOL BREF scale. These variables were then utilized in multivariate regression analysis

Among the 16 variables utilized for multivariate logistic regression analysis, 8 variables (age, educational status, marital status, occupation, residence, history of comorbidity, family support and recreational activity) stated as on (Table -5) were identified to be associated with QOL through backward LR methods of multivariate binary logistic regression. 
Table 5

Association factors of quality of life among those patients treated for epilepsy at Dessie referral hospital, Ethiopia, 2020

\begin{tabular}{|c|c|c|c|c|c|c|}
\hline \multirow[t]{2}{*}{ Variables } & \multirow[t]{2}{*}{ Category } & \multicolumn{2}{|c|}{$\begin{array}{l}\text { Health relate } \\
\text { quality of life }\end{array}$} & \multirow[t]{2}{*}{$\begin{array}{l}\text { COR of } 95 \% \\
\mathrm{Cl}\end{array}$} & \multirow[t]{2}{*}{ AOR } & \multirow[t]{2}{*}{$\begin{array}{l}P \text { - } \\
\text { Value }\end{array}$} \\
\hline & & Poor & Good & & & \\
\hline \multirow[t]{4}{*}{ Age } & $18-24$ years & 61 & 44 & 1 & 1 & 0.001 \\
\hline & $25-34$ years & 65 & 46 & $\begin{array}{l}0.981(0.571, \\
1.686)\end{array}$ & $\begin{array}{l}0.409(0.192 \\
0.875)\end{array}$ & 0.021 \\
\hline & $35-44$ years & 25 & 45 & $\begin{array}{l}2.495(1.337 \\
4.657)\end{array}$ & $\begin{array}{l}2.101(0.748 \\
5.906)\end{array}$ & 0.159 \\
\hline & $>44$ years & 56 & 42 & $\begin{array}{l}1.040(0.596, \\
1.815)\end{array}$ & $\begin{array}{l}0.914(0.33 \\
2.477)\end{array}$ & 0.860 \\
\hline \multirow[t]{5}{*}{$\begin{array}{l}\text { Educational } \\
\text { status }\end{array}$} & $\begin{array}{l}\text { Unable to read } \\
\text { and write }\end{array}$ & 69 & 40 & 1 & 1 & 0.001 \\
\hline & Elementary & 57 & 41 & $\begin{array}{l}1.241(0.709 \\
2.171)\end{array}$ & $\begin{array}{l}0.502(0.224 \\
1.124)\end{array}$ & 0.094 \\
\hline & Secondary & 45 & 31 & $\begin{array}{l}1.188(0.652 \\
2.167)\end{array}$ & $\begin{array}{l}0.167(0.061 \\
0.455)\end{array}$ & 0.000 \\
\hline & Diploma & 23 & 29 & $\begin{array}{l}2.175(1.111, \\
4.258)\end{array}$ & $\begin{array}{l}0.397(0.121 \\
1.300)\end{array}$ & 0.127 \\
\hline & $\begin{array}{l}\text { Degree and } \\
\text { Above }\end{array}$ & 13 & 36 & $\begin{array}{l}4.777(2.269 \\
10.055)\end{array}$ & $\begin{array}{l}0.984(0.251, \\
3.860)\end{array}$ & 0.981 \\
\hline \multirow[t]{4}{*}{ Marital status } & single & 94 & 60 & 1 & 1 & 0.001 \\
\hline & Married & 67 & 108 & $\begin{array}{l}2.525(1.619 \\
3.940)\end{array}$ & $\begin{array}{l}2.080(1.014 \\
4.267)\end{array}$ & 0.046 \\
\hline & Divorce & 29 & 9 & $\begin{array}{l}0.486(0.215 \\
1.098)\end{array}$ & $\begin{array}{l}0.295(0.092 \\
0.950)\end{array}$ & 0.041 \\
\hline & Widowed & 17 & 0 & $0.000(0.000)$ & $0.000(0.000)$ & 0.998 \\
\hline \multirow[t]{5}{*}{ occupation } & Unemployed & 69 & 40 & 1 & 1 & 0.037 \\
\hline & Famer & 57 & 41 & $\begin{array}{l}1.241(0.709 \\
2.171)\end{array}$ & $\begin{array}{l}0.605(0.224 \\
1.633)\end{array}$ & 0.321 \\
\hline & House wife & 45 & 31 & $\begin{array}{l}1.188(0.652, \\
2.167)\end{array}$ & $\begin{array}{l}3.759(0.905 \\
15.61)\end{array}$ & 0.068 \\
\hline & Employed & 23 & 29 & $\begin{array}{l}2.175(1.111 \\
4.258)\end{array}$ & $\begin{array}{l}2.284(0.831 \\
6.272)\end{array}$ & 0.109 \\
\hline & Self-employed* & 13 & 36 & $\begin{array}{l}4.777(2.269 \\
10.055)\end{array}$ & $\begin{array}{l}2.155(1.001, \\
4.638)\end{array}$ & 0.050 \\
\hline
\end{tabular}




\begin{tabular}{|c|c|c|c|c|c|c|}
\hline \multirow[t]{2}{*}{ Residence } & Rural & 136 & 48 & 1 & 1 & \\
\hline & Urban & 71 & 129 & $\begin{array}{l}5.148(3.321, \\
7.980)\end{array}$ & $\begin{array}{l}\text { 7.074(3.573, } \\
14.005)\end{array}$ & 0.000 \\
\hline \multirow{2}{*}{$\begin{array}{l}\text { History of } \\
\text { comorbidity }\end{array}$} & No & 170 & 164 & 1 & 1 & \\
\hline & Yes & 37 & 13 & $\begin{array}{l}2.746(1.409 \\
5.351)\end{array}$ & $\begin{array}{l}3.566(1.298 \\
9.798)\end{array}$ & 0.014 \\
\hline \multirow[t]{2}{*}{ Family support } & No & 16 & 2 & 1 & 1 & \\
\hline & Yes & 191 & 175 & $\begin{array}{l}0.136(0.31 \\
.602)\end{array}$ & $\begin{array}{l}9.275(1.073, \\
80.176)\end{array}$ & 0.043 \\
\hline \multirow{2}{*}{$\begin{array}{l}\text { Recreational } \\
\text { activity }\end{array}$} & No & 83 & 38 & 1 & 1 & \\
\hline & Yes & 124 & 139 & $\begin{array}{l}0.364(0.187 \\
0.71)\end{array}$ & $\begin{array}{l}2.229(1.184, \\
4.196)\end{array}$ & 0.013 \\
\hline
\end{tabular}

\section{Discussion}

In developing countries the morbidity of epilepsy was increased significantly including Ethiopia. The increased population in developing countries affects the health system and adds socioeconomic burden requiring urgent attention. Many people in Africa believe that epilepsy is contagious; because of these unwilling to help or to touch the person who has fallen during a seizure lead to enormous social stigmas and lower QOL (3). Epilepsy affects life in multi-faced ways which should be studied in every community's cultural conditions and psycho social makeup. It is to be framed within the literature considering quality of life has an important role in the disease management quality outcome (14), (15). In order to measure success of the services delivery system that patient's quality of life is essential to provide opportunity for improvement of health services since feedback from patient side is a bench mark for health care quality improvement and continued the treatment protocol.

The present study reveals that the mean score overall level of epileptic patient's quality of life treated at out-patient psychiatry clinic of Dessie referral hospital were 51.98 (95\% Cl:41.9-62.06) and this study was aligned studies done in Uganda and Kenya 58 and 49.9 respectively (16), (17). It was also aligned studies done in Ethiopia at Jimma University Medical Center (58.8) (7), Addis Ababa Amanuel Mental Specialized Hospital were 56.36 (3), Ambo general hospital (56.43) (5), Public hospitals of Wollega zones (60.47) (18), but it was differed studies done in Mekelle City (77.97\%) (2). This may be due to research done in Mekelle city was used QOLIE-31 tool that was focus on the seizure worry, wellbeing, energy/fatigue, cognitive functioning and medication effect. This finding also lower than studies done in Brazil (68.73) (11) and Malaysia (68.9) (8). This difference might be due to those countries had better quality of health services, good socioeconomic status of the population than Africa population. On the other hand in Africa including the present study population there was low socioeconomic status and the number and level of the health care institutions was less when compared to the population size; that may be affected the quality of life for PLWE. 
On this findings quality of life on physical domain mean ranked relatively higher (58.6) in the WHOQOLBRIEF scaled scores when compared to the social (53.3), psychological (50.25\%) and environmental domain mean (48.75), however the finding of this study on physical domain mean was lower than studies done in Brazil (72.76) (11). This might be due to the management approach for epilepsy in this hospital might not be focus on adopting a holistic approach that also incorporates their psychological, social and environmental needs rather than focusing only seizure control.

The finding shows that the mean values of psychological domain mean of WHOQOL-BRIEF scale were 50.25 like the meaningfulness of life, the capacity of concentrating on daily activity the ability of accepting their body appearance and environmental domain mean were 48.75; those were healthy condition of living environment, the availability of information about their daily life and the accessibility of health service including transportation, are lower than the physical domain mean 58.6 and social domain mean 53.3. This difference may be due to most of the study participant marital status was single; their educational level was also unable to read and write and most of study participant had no job (unemployed) those factors might had been effect on the psychological perspective of epileptic patients. On the other hand $12.2 \%$ and $37 \%$ of study participant had depression and anxiety respectively this might had their own contribution on the psychological domain of WHOQOL-BRIEF scale. For environmental domain almost half of (47.9\%) the study participant were live in rural area, that may be affected the healthy condition of living environment, the availability of information about their daily life and the accessibility of health service and transportation.

Educational status had negatively association for good quality of life those patients who had completed Secondary school were $83.3 \%$ less likely good quality of life when compared to unable to read and write patients (AOR $=0.167,95 \% \mathrm{Cl}: 0.061,0.455)$. But other studies done in Uganda, Kenya, Georgia shows that high educational level had strongly associated with good quality of life scores (16), (17), (19). In Ethiopia also studies done in Amanuel Mental Specialized Hospital, Ambo General Hospital and Mekelle city high education was associated with good quality of life in epileptic patient (3), (5), (2). This might be due educated patients had good understand about the natural disease history of epilepsy and they were good adherence about the treatment. Current study reviles that Age of the participant had association for good quality of life those patients age group between $25-34$ years were about $59.1 \%$ less likely to have good quality of life as age group between $18-24$ years $(\mathrm{AOR}=0.409,95 \% \mathrm{Cl}: 0.192,0.875)$. This study was aligned studies done in Malaysia, Italy and Georgian (8), (20), (19).

Occupational status had association for good quality of life. Patients who had self-employed was 1.155 times more likely good quality of life when compared to unemployed ( $\mathrm{AOR}=2.155,95 \% \mathrm{Cl}$ : 1.001, 4.638). This studies was supported by studies done in Kenya (17) and in Ethiopia at Jimma University Medical Center (7). This might be due to patients who had job can be cover their treatment cost, transportation and other health related costs by themselves. The second justification employed patients had also relatively enough money to meet their daily needs when compared to unemployed patients. 
The present research was indicated that patients who were living in urban area had 7.07 times more likely to have good quality of life as compared to patient who were living in rural area $(\mathrm{AOR}=7.074,95 \% \mathrm{Cl}$ :

$3.573,14.005)$. This study was aligned studies done in Kenya (17) and studies done in Ethiopia at Jimma University Medical Center (7). This might be due to patients who had live in rural area can be unable to get completed information about their health status, access health institutions nearby their location, and they cannot be get the opportunity to participate in leisure activities.

Marital status was association for good quality of life those patients who were married had 2.5 times more likely to have good quality of life (AOR $=2.525,95 \% \mathrm{Cl}: 1.619,3.940)$ as compared to patient who were single. This study was similar study done in Uganda (16). The possible reasons of this difference may be married patients had stable life condition. They can be get psychological support from their partners and can be feel comfortable about their personal relationships.

History of co-morbid illness was another factor associated with quality of life. Those who had no history of co-morbid illness had 3.566 times more likely good quality of life as compared to patients who had history of comorbid illness (AOR $=3.566,95 \% \mathrm{Cl}$ : $1.298,9.798)$. This study was aligned to studies done in India (1). In Ethiopia also studies done at Jimma University Medical Center, Ambo General Hospital and public hospitals of Wollega zones indicates that absence of comorbid illness were good predicators of good quality of life (7) (5), (18). The possible reasons of this difference adopted from different literatures patients who had no comorbid illness like depression, anxiety, cognitive impairments, and psychosocial issue were good quality of life as compared to patients who had comorbid illness.

Patients who had family support had 9.275 times more likely good quality of life (AOR $=9.27595 \% \mathrm{Cl}$ : $1.073,80.176)$ than patients who had no family support on their life. On the other hand epileptic patients participating on recreational activity had 2.229 times more likely good quality of life ( $A O R=2.229,95 \% \mathrm{Cl}$ : 1.184, 4.196). Different literatures indicate personal characteristics like self-esteem, family support, doing regular physical activity and participating on recreational activities were strong predictors of good quality of life. This might be due to persons who had good personal character can be the capacity of avoiding negative feelings on their personal life, concentrate on their daily activities, enjoy with their life and they can be made their life meaningful.

\section{Conclusion And Recommendation}

The mean overall quality of life of epileptic patients at Dessie referral hospital was low. The finding is supported by low quality of service on environmental domain. In multivariate linear regression analysis; from socio-demographic characteristics age, educational status, marital status, occupation and residence; from clinical characteristics history of comorbidity and from personal characteristics family support and recreational activity was associated with quality of life in epileptic patient. In general, a concerted effort must be made to improve quality of life for epileptic patient at Dessie referral hospital in Ethiopia. 


\section{Limitation Of The Study}

The cross-sectional design of this study limited the determination of causal relationships and was time based.

\section{Abbreviations}

AED- Anti-Epileptic Drug, AOR-Adjusted Odds Ratio, Cl- Confidence Interval, CNC- Central Nervous System, HDAS- Hospital Depression \& Anxiety Scale, HRQOL- Health Related Quality of Life, PLWE- People living With Epilepsy, QOL- Quality of Life, OPD- Out-Patient Department, SPSS- Statistical Package for Social Sciences, WHO-QOL BRIEF -World Health Organization Quality of Life Instrument, WHOQOL- World Health Organization Health Related Quality of Life, RX-Treatment.

\section{Declarations}

\section{Ethical consideration}

Ethical clearance was obtained from ethical review committee of College of Medicine \& health Sciences, Wollo University. The formal permission letter was obtained from Dessie Referral Hospital administration. Before data collection the purpose and importance of the study was explained to each study participant. For the purpose of obscurity, informed was obtained and participant's name was not be used at the time of data collection and all other personnel information kept entirely obscure and confidentiality was assured throughout the study period.

\section{Consent for publication}

Not applicable

\section{Availability of data and materials}

All relevant data are within the manuscript and its Supporting Information files.

\section{Competing interests}

We declare no competing interests.

\section{Funding}

Funding was not received from any organization and the authors are from low income country 


\section{AKNOWLEGMENT}

The authors would like to thank all the study participants for their time and willingness to participate in the study. We would also like to forward our sincere gratitude to the Dessie referral hospital epilepsy outpatient department staff members and the data Collectors for their support throughout the study period. We would like also to thanks Wollo University, college of medicine and health science, school of nursing and midwifery, department of adult nursing for giving us the chance to doing this article.

\section{Authors' contributions}

ASS designed the study, participated in and monitored the data collection process, analysed the data, and wrote the first draft of the manuscript. Dr. PK Participated in the design, reviewing and modifying the drafts of the manuscript. DAB also involved in first draft writing, reviewing and analysis of the drafts of the manuscript. The rest author's had equal contribution in the design and data collection stages of the study. All the authors have read and approved the final version of the manuscript.

\section{Authors' information}

ASS has MSc in adult nursing, Dr. PK has PhD supervisor and consultant, DAB has BSc in Pharmacy. SLH has MPH in human nutrition, YSA has MPH in human nutrition.

\section{References}

1. Shanmukhi S, Jayalakshmi S, Anand B. Factors Associated with Quality of Life in Adult Epilepsy Patients: a Hospital Based Study from South India. Res Neurol An Int J. 2015;2015:1-9.

2. Gebre AK, Haylay A. Sociodemographic, clinical variables, and quality of life in patients with epilepsy in Mekelle City, Northern Ethiopia. Behav Neurol. 2018;2018.

3. Awoke AA, Mossie TB, Yesigat MA. Assessment of quality of life and associated factors among people with epilepsy attending at Amanuel Mental. Sci J Public Heal. 2014;2(5):378-83.

4. WHO I, Against Epilepsy League IB for E. EPILEPSY A public health imperative. 2019.

5. Tefera GM, Megersa WA, Gadisa DA. Health-related quality of life and its determinants among ambulatory patients with epilepsy at Ambo General Hospital, Ethiopia: Using WHOQOL-BREF. PLoS One. 2020;15(1):1-16.

6. INTRODUCTION , ADMINISTRATION, SCORING AND GENERIC VERSION OF THE ASSESSMENT Field Trial Version December 1996 PROGRAMME ON MENTAL HEALTH WORLD HEALTH ORGANIZATION. 1996;(December).

7. Shiferaw D, Hailu E. Quality of Life Assessment among Adult Epileptic Patients Taking Follow Up Care at Jimma University Medical Center, Jimma, South West Ethiopia: Using Quality of Life in Epilepsy Inventory-31instrument. Glob Journals. 2018;18(3). 
8. Norsa'adah B, Zainab J, Knight A. The quality of life of people with epilepsy at a tertiary referral centre in Malaysia. Health Qual Life Outcomes. 2013;11(1):9-11.

9. Tiki1 $\mathrm{T},{ }^{*} \mathrm{AB}$ and $\mathrm{KM}, 1 \mathrm{Ambo}$. Assessment of the Prevalence and Factors Associated to Perceived Stigma among Epileptic Patient who are on Follow up at Hospitals in West Shewa. J Neurol Neurobiol. 2018;4(2):1-6.

10. Sinha A, Sanyal D, Mallik S, Bengal W, Sengupta P. Annals of Indian Academy of Neurology. 2011; (March).

11. Siqueira NF, Oliveira FLBB, Siqueira JA, De Souza EAP. Quality of life in epilepsy: A study of Brazilian adolescents. PLoS One. 2014;9(9).

12. Kassahun G, Moges G, Demessie Y. Assessment of patients'adherence to antiepileptic medications at dessie referral hospital,chronic follow-up, south wollo,amhara region,north east Ethiopia. Neurol Res Int. 2018;2018.

13. HADS). Hospital Anxiety and Depression Scale ( HADS ). (D):21.

14. M ARCIA A. T ESTA, M.P.H., P H .D., AND D ONALD C. S IMONSON MD. CURRENT CONCEPTS on ASSESS ASSESSMENT OF QUALITY-OF-LIFE OUTCOMES M Review articles. Curr CONCEPTS. 1996;334(13):835-40.

15. Karimi M. Health , Health-Related Quality of Life, and Quality of Life: What is the Difference? Pharmacoeconomics. 2017;(October).

16. Nabukenya AM, Matovu JKB, Wabwire-Mangen F, Wanyenze RK, Makumbi F. Health-related quality of life in epilepsy patients receiving anti-epileptic drugs at National Referral Hospitals in Uganda: A cross-sectional study. Health Qual Life Outcomes [Internet]. 2014;12(1):1-8. Available from: Health and Quality of Life Outcomes

17. Kinyanjui DWC, Kathuku DM, Mburu JM. Quality of life among patients living with epilepsy attending the neurology clinic at kenyatta national hospital, Nairobi, Kenya: A comparative study. Health Qual Life Outcomes [Internet]. 2013;11(1):1. Available from: Health and Quality of Life Outcomes

18. Abadiga M, Mosisa G, Amente T, Oluma A. Health-related quality of life and associated factors among epileptic patients on treatment follow up at public hospitals of Wollega zones, Ethiopia, 2018. BMC Res Notes [Internet]. 2019;12(1):1-7. Available from: https://doi.org/10.1186/s13104-0194720-3

19. Djibuti M, Shakarishvili R. Influence of clinical, demographic, and socioeconomic variables on quality of life in patients with epilepsy: J Neurol Neurosurg Psychiatry. 2003;74:570-3.

20. Edefonti V, Bravi F, Turner K, Beghi E, Canevini MP, Ferraroni M, et al. Health-related quality of life in adults with epilepsy: The effect of age, age at onset and duration of epilepsy in a multicentre Italian study. BMC Neurol [Internet]. 2011;11(1):33. Available from: http://www.biomedcentral.com/1471$2377 / 11 / 33$

\section{Figures}




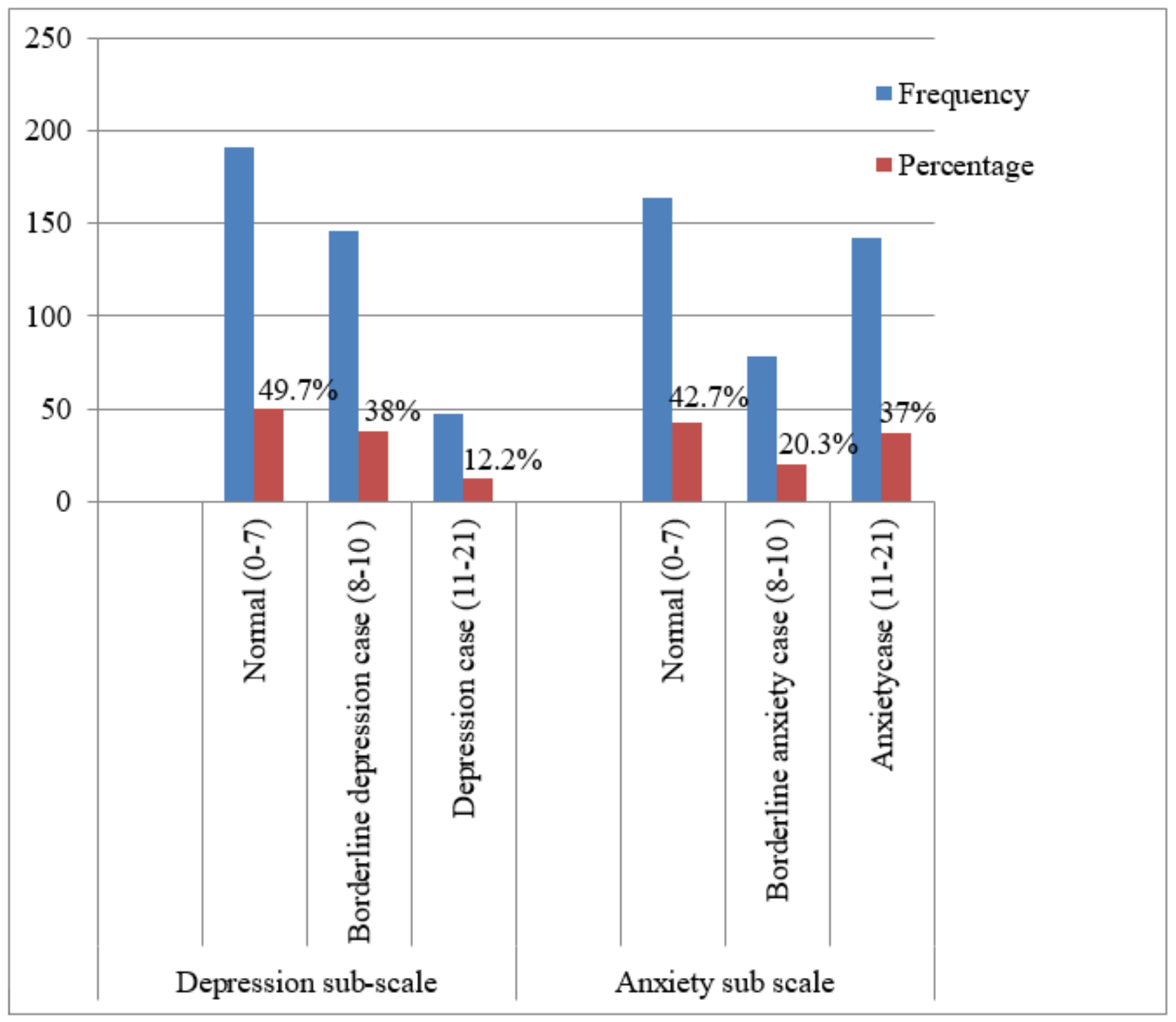

Figure 1

Hospital depression and anxiety scale of study participant, Dessie referral hospital, Ethiopia, 2020 